\title{
Evaluation of Medium Access and a Positioning System in Wireless Underground Sensor Networks
}

\author{
Sérgio Conceição \\ INESC TEC, \\ Universidade do Porto \\ sdlc@inesctec.pt
}

\author{
Cristiano Pendão \\ Centro de Investigação Algoritmi, \\ Universidade do Minho \\ cpendao@dsi.uminho.pt
}

\author{
Adriano Moreira \\ Centro de Investigação Algoritmi, \\ Universidade do Minho \\ adriano.moreira@algoritmi.uminho.pt
}

\author{
Manuel Ricardo \\ INESC TEC, \\ Universidade do Porto \\ mricardo@inesctec.pt
}

\begin{abstract}
Wireless Underground Networks (WUN) have many applications, such as border surveillance, agriculture monitoring, and infrastructure monitoring. Recent studies have shown that they are feasible and have deployment advantages over wired networks, but only a few WUN evaluations in multiple access scenarios have been done. This paper presents a simulation study on medium access for a WUN with 4 nodes buried, and one node aboveground. The simulations were carried out using the ns-3 simulator and they evaluate both Wi-Fi, and Lr-Wpan networks for dry and wet soils. We verified that for the same number of concurrent nodes, the use of the RTS/CTS mechanism has a much higher influence than the soil water content. Furthermore, a study about the feasibility of using Wi-Fi fingerprinting for positioning above the ground based on the buried infrastructure revealed promising results.
\end{abstract}

Keywords-Wireless Underground Networks, Wi-Fi Positioning, Medium Access, Simulation.

\section{INTRODUCTION}

A wireless underground network includes nodes underground monitoring some physical properties, and nodes aboveground acting as data sinks or control nodes. This type of scenarios leads to communication links between underground nodes and links between underground and aboveground nodes; in the latter case, a hybrid propagation medium is in place (air plus soil). In addition, propagation in the composite underground-aboveground channel is not symmetric [1]; in the underground to aboveground direction the attenuation is lower due to the fact that in this case the electromagnetic signal travels from a medium with a higher refractive index (soil) to a medium with lower refractive index (air).

The first contribution of this paper is the comparative study of alternative medium access mechanisms in the underground and composite media, done through simulation. The simulations where done for different number of concurrent nodes, dry and wet soil, and both with and without the RTS/CTS mechanism. The second contribution is the demonstration of the feasibility of using buried nodes for building a Wi-Fi radio map that can be used for localization purposes.

Positioning based on Wi-Fi fingerprinting involves two phases: a calibration (offline) phase, and a positioning (online) phase. In the calibration phase samples about the surrounding radio environment are collected and annotated with the corresponding geometric position; one or a group of these samples for a specific position are known as a fingerprint, and the set of all fingerprints collected over the coverage area of the positioning system is known as the radio map. In the positioning phase, the device collects a sample of the surrounding radio environment (a fingerprint) that is then used to estimate the device's position by comparing it to the fingerprints in the radio map [2].

This paper is organized as follows. Section II introduces the underground propagation medium. Section III defines the methodology followed to evaluate the underground network. Section IV presents the results obtained. Finally, Section V draws the major conclusions, and points out directions for future work.

\section{UNDERGROUND MEDIUM AND WUN}

A typical WUN includes buried nodes and aboveground nodes acting as data sinks. Section II-A introduces application scenarios for WUN, and Section II-B presents the propagation models for estimating the attenuation in these links.

\section{A. Application Scenarios}

Agriculture is one of the most promising scenarios for these networks. In this case, sensors can be used to monitor the soil parameters, such as water content, mineral content, salinity, and temperature, and then communicate these values in real time to a control station aboveground, so that irrigation can be controlled automatically, as described in [3]. The same irrigation control mechanisms can also be applied to assist the maintenance of sport fields such as golf courses.

Security is an area that may also benefit from the deployment of underground networks due to the concealment of the nodes. The sensors can be buried at a shallow depth and detect movement at the surface. This is useful for home security as well as for military applications such as border patrol [4].

Infrastructure monitoring is another possible application scenario for WUN. In this case a WUN can be deployed, for instance, to monitor pipelines and detect leakages. This is proposed in [5], and different deployments are highlighted such as using the sensors inside or outside the pipeline.

\section{B. Electromagnetic Propagation Models}

In this section the EM propagation models for soil communications are presented. Although these models can be used to all soil types a proper characterization of the soil dielectric constant $(\epsilon)$ is required. This constant has a major dependency with soil water content, meaning that the EM 
waves propagation are highly dependent on Volumetric Water Content (VWC).

1) Underground-to-underground Scenario: The propagation model used for the underground-to-underground (U2U) scenario is the 3-ray model [6]. This propagation model considers three propagation waves - direct wave, reflected wave, and lateral wave. Fig. 1 illustrates the three waves.

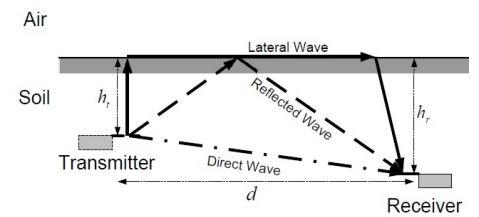

Fig. 1. The three electromagnetic waves. [6]

As we can observe from Fig. 1, the lateral wave propagates in the soil, then in the air, and finally back in the soil. Since the attenuation in the air is lower than in the soil, the lateral wave is the dominant component for lower depths and high horizontal distances. From [6] the received power from each wave can be calculated as:

$$
\begin{array}{cc}
P_{d}= & P_{t}+20 \log \left(\lambda_{s}\right)-20 \log \left(r_{1}\right)- \\
& -8.69 \alpha r_{1}-45 \\
P_{r}= & P_{t}+20 \log \left(\lambda_{s}\right)-20 \log \left(r_{2}\right)- \\
& -8.69 \alpha r_{2}+20 \log (\Gamma)-45 \\
P_{l}= & P_{t}+20 \log \left(\lambda_{s}\right)-40 \log (d)- \\
& -8.69 \alpha\left(h_{t}+h_{r}\right)+20 \log (T)-30
\end{array}
$$

where $P_{d}, P_{r}$ and $P_{l}$ are respectively the powers received (in $\mathrm{dBm}$ ) from the direct wave, reflected wave and lateral wave. $P_{t}$ is the transmitter power, $\lambda_{s}$ is the wavelength in the soil, $r_{1}$ and $r_{2}$ are the distances traveled, respectively, by the direct ray and the reflected ray, $d$ is the horizontal distance, $\Gamma$ is the reflection coefficient, $\alpha$ is the attenuation constant, $T$ is the refraction coefficient, and $h_{t}$ and $h_{r}$ are respectively the depths of the transmitter and receiver. The total power received (in $\mathrm{dBm}$ ) consists of the three components presented in Eq. 4.

$$
P_{r e c}=10 \log \left(10^{\frac{P_{d}}{10}}+10^{\frac{P_{r}}{10}}+10^{\frac{P_{l}}{10}}\right)
$$

2) Hybrid Scenario: In this scenario one of the nodes is buried in the soil and the other is located aboveground, so the propagation medium is hybrid (soil plus air). The direction of communication is important because the links are asymmetric. In fact, the Underground to Aboveground (U2A) direction has lower attenuation than the Aboveground to Underground (A2U). This is explained by the fact that in the $\mathrm{A} 2 \mathrm{U}$ direction the wave is propagating from a low refractive index to a higher refractive index, which leads to higher reflections and, consequently, less energy propagating through soil. Also, according to [7] the aboveground path is associated to a multipath fading channel that can be modeled as a Rayleigh distributed random variable $\chi$. The probability density function is defined by Eq. 5:

$$
f(\chi)=\frac{\chi}{\sigma_{R}^{2}} e^{-\chi^{2} /\left(2 \sigma_{R}^{2}\right)}
$$

where $\sigma_{R}$ is the Rayleigh distribution parameter derived from field experiments in the deployment environment.

According to [7] the path loss for the hybrid scenario can be calculated using the following equations:

$$
\begin{aligned}
L_{x-y} & =L_{u}+L_{a}+L_{\text {int }-y}-10 \log \left(\chi^{2}\right) \\
L_{u} & =6+20 \log \left(d_{s}\right)+20 \log (\beta)+8.69 \alpha d_{s} \\
L_{a} & =20 \log (f)+20 \log \left(d_{a}\right)-147.56
\end{aligned}
$$

From Eq. 6 we can observe that the attenuation $\left(L_{x-y}\right)$ is the sum of four components, where $L_{u}$ is the attenuation in the soil, $L_{a}$ is the attenuation in the air, $L_{i n t x-y}$ is the attenuation in the interface between soil and air, and $-10 \log \left(\chi^{2}\right)$ is the attenuation associated with the multipath fading.

If we consider the $\mathrm{U} 2 \mathrm{~A}$ direction the interface is soil-air. In this case the losses in the interface $\left(L_{\text {intx-y }}\right)$ are estimated by Eq. 9:

$$
L_{u g-a g} \approx 10 \log \frac{\left(\sqrt{\epsilon^{\prime}}+1\right)^{2}}{4 \sqrt{\epsilon^{\prime}}}
$$

On the other hand, if the direction is A2U the interface is air-soil, and the losses in the interface $\left(L_{i n t x-y}\right)$ are given by Eq. 10:

$$
L_{a g-u g} \approx 10 \log \frac{\left(\cos \theta_{i}+\sqrt{\epsilon^{\prime}-\sin ^{2} \theta_{i}}\right)^{2}}{4 \cos \theta_{i} \sqrt{\epsilon^{\prime}-\sin ^{2} \theta_{i}}}
$$

\section{Methodology}

In this section we detail the network topology that was simulated, and the simulation setups that were used. Fig. 2 illustrates the basic scenario that consists on one node placed aboveground at coordinates $(0 ; 0 ; 1.0)$, and four nodes buried at coordinates $(-30.0 ; 0 ;-0.1),(0 ;-30.0 ;-0.1),(30.0 ; 0 ;-0.1)$, and $(0 ; 30.0 ;-0.1)$, all the values represented in meters $(\mathrm{m})$. These locations were chosen so that all buried nodes are horizontally separated $30 \mathrm{~m}$ from the aboveground node, meaning that each underground node can always reach the aboveground node but cannot sense the other underground nodes due to the higher path loss in the U2U channel.

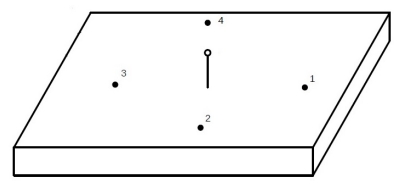

Fig. 2. Simulation scenario

The soil used for the simulations is characterized by the following parameters: $17 \%$ sand, $28 \%$ of clay, particle density of $2.66 \mathrm{~g} / \mathrm{cm}^{3}$, and bulk density of $1.3 \mathrm{~g} / \mathrm{cm}^{3}$. The simulations were done for a dry soil, with $10 \%$ VWC, and a wet soil, with $25 \%$ VWC. 
In terms of network technologies, we used the IEEE 802.15.4 standard for wireless sensor networks (Lr-Wpan), and the IEEE 802.11g standard for Wi-Fi, both operating in the $868 \mathrm{MHz}$ frequency band. On top of the 802.15.4 standard the IETF 6lowpan standard was used in order to enable the use of IPv6 in the Lr-Wpan simulations.

\section{A. Evaluation of Medium Access Mechanisms in WUN}

In order to evaluate the medium access mechanisms, two simulation setups were considered. The first setup was used to evaluate the medium access mechanisms of an underground Wi-Fi network (802.11g), while the other setup was used to evaluate an underground Lr-Wpan (802.15.4) network. Both setups use the same positions for the nodes, and the same operating frequency $(868 \mathrm{MHz})$. The parameters that differ from one setup to the other are shown in Table I.

TABLE I. SIMUlation PARAMETERS.

\begin{tabular}{lcr}
\hline Parameter & Lr-Wpan & Wi-Fi \\
\hline Transmission power $(\mathrm{dBm})$ & 0 & 20 \\
Transmitter antenna gain $(\mathrm{dBi})$ & 0 & 2.0 \\
Receiver antenna gain $(\mathrm{dBi})$ & 0 & 3.0 \\
Receiver Sensitivity $(\mathrm{dBm})$ & -106 & -90 \\
Packet size (bytes) & $32,64,120$ & 1024,1536 \\
Data Rate (kbit/s) & 10 & 1024 \\
Simulation Time (s) & 600 & 60 \\
\hline
\end{tabular}

The simulations were performed for dry and wet soils in order to evaluate the differences in performance for different VWC. The number of nodes were varied, in order to detect the influence of having a different number of nodes competing for the medium. Furthermore, the RTS/CTS mechanism was also tested in Wi-Fi simulations to determine if it performs as expected for underground networks, and also to evaluate the situations when it is required (i.e. the situations where the network performance drops drastically if the RTS/CTS is not used). For Lr-Wpan this mechanism was not tested because ns-3 still does not offer an implementation of RTS/CTS for Lr-Wpan networks.

The traffic was generated by the underground nodes, using the ns-3 OnOff application, and the aboveground node was configured to use the ns-3 application DataSink, in order to collect the traffic of all the underground nodes. To analyze the network performance the ns-3 flow monitor was used and configured to collect the network throughput, packet delivery ratio, and packet delay. These metrics provide an overview of the overall network performance.

\section{B. Evaluation of the positioning system}

To evaluate the feasibility of the fingerprinting-based positioning system on the soil surface, a set of RSSi samples from several points had to be collected. Each sample contains the measured RSSi and MAC address of each detected node, and the position where the sample was obtained. This position needs to be as accurate as possible in order to allow using the collected samples as a radio map.

Since the soil water content has a large influence in the RSSi, two simulation setups were configured to obtain the RSSi fingerprints, one simulation for dry soil, and another for wet soil. A set of 12 reference points was defined to measure the RSSi. All the samples were obtained at a $10 \mathrm{~cm}$ height, in order to avoid the interface soil-air. The position of each reference point is shown in Fig. 3.

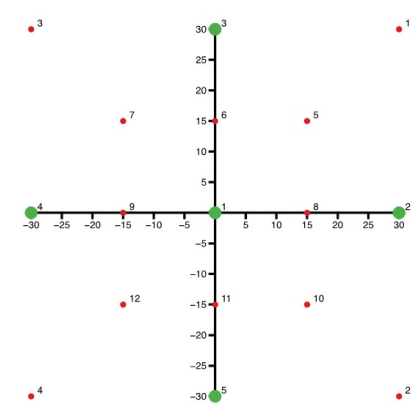

Fig. 3. Location of the 12 reference points (in red).

Another RSSi data collection was made in order to obtain a more uniform radio map. In this new collection, another set of measured points were added to the 12 points already measured in the first setup (Fig. 3), so that each measure is separated horizontally, and vertically from each other by 15 meters. Fig. 4 shows all the points that were used to collect the RSSi points. In this case the RSSi values were collected only for the dry soil $(10 \%$ VWC).

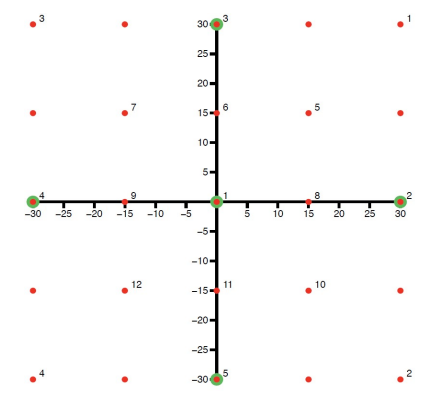

Fig. 4. Location of all the reference points (in red).

The location of the reference points was chosen in order to provide a radio map that was based on a grid. This allowed us to randomly position a set of nodes in the simulated terrain (using the ns-3 RandomBoxPositionAllocator), and for each point compare its RSSi with the RSSi from the measured points, and choose the location of the random point as the location of the measured point with the closer RSSi values (1-nearest neighbour approach). The comparison between the RSSi values of the random point and the RSSi of a radio map sample was measured using the Euclidean distance between the two readings $\left(\sqrt{\left(R S S i_{1}^{A}-R S S i_{1}^{B}\right)^{2}+\left(R S S i_{2}^{A}-R S S i_{2}^{B}\right)^{2}+\ldots}\right)$.

\section{REsults}

This section presents and discusses the results obtained by the simulation setups presented in Section III. Subsections IV-A and IV-B present the results relative to the medium access mechanisms respectively for the Lr-Wpan and Wi-Fi networks. Subsection IV-C that presents the results for the positioning system simulations. 


\section{A. Results for Lr-Wpan simulation}

The first simulation setup for Lr-Wpan consisted in evaluating the network performance dependency on the VWC. The simulations were carried out for 2,3 , and 4 nodes buried, and repeated for dry (10\% VWC) and wet (25\% VWC) soils. Fig. 5, 6, and 7 present the obtained results for the throughput, packet delivery ratio (PDR), and delay, respectively. The confidence intervals represented are calculated for a $95 \%$ confidence level.

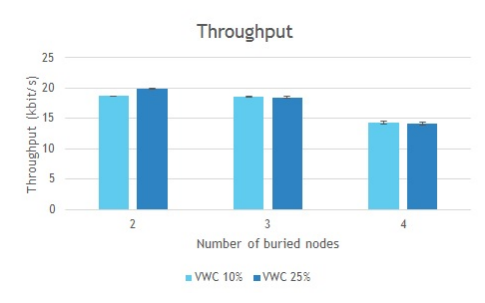

Fig. 5. Throughput for Lr-Wpan network for dry and wet soils.

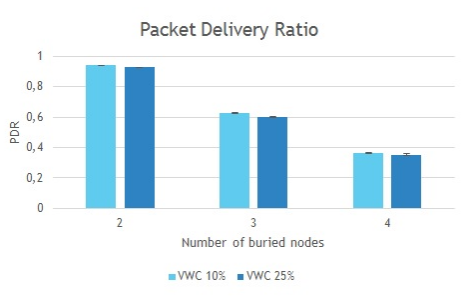

Fig. 6. PDR for Lr-Wpan network for dry and wet soils.

From Fig. 5, and 6 we conclude that the VWC has a negligible effect on the throughput and PDR. The major impact is on the number of concurrent nodes accessing the medium, that makes the PDR to decrease from $94 \%$ for 2 nodes, to $62 \%$ for 3 nodes, and to $33 \%$ for 4 nodes. From these values we conclude that the network is operable without the RTS/CTS mechanism if it has 3 or less nodes. Otherwise the PDR drops below $50 \%$, and the network becomes highly unreliable.

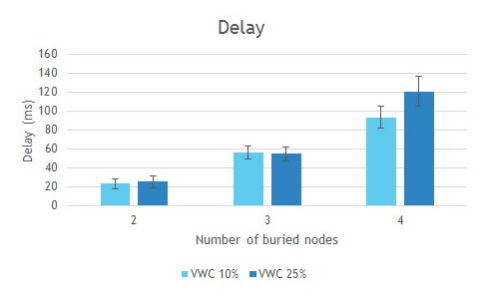

Fig. 7. Delay for Lr-Wpan network for dry and wet soils.

For the delay, the number of nodes continues to have the major impact on the network performance, since the delay increases from $11 \mathrm{~ms}$ for 2 nodes, to $19 \mathrm{~ms}$ for 3 nodes, and to $38 \mathrm{~ms}$ for 4 nodes (dry soil). Nevertheless, the VWC has some impact on the packet delay, specially for the 4 nodes case where the delay increases from $38 \mathrm{~ms}$ for the dry soil to $43 \mathrm{~ms}$ for the wet soil.

For the second simulation setup the soil was dry, and the purpose was to evaluate the network performance for different packet sizes, and for a different number of nodes. Fig. 8, and
9 present the results obtained respectively for the PDR and delay.

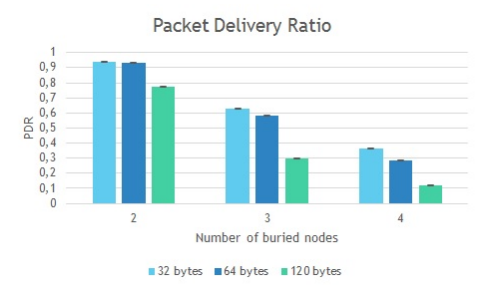

Fig. 8. PDR for Lr-Wpan network for dry soil.

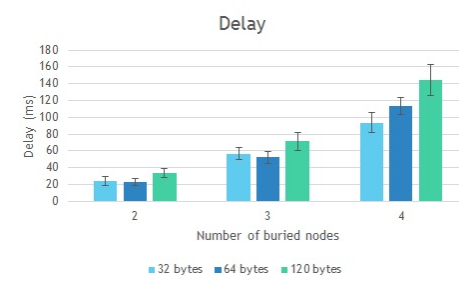

Fig. 9. Delay for Lr-Wpan network for dry soil.

From Fig. 8 we conclude that the PDR is almost identical for 32 and 64 bytes packet length, and it drops from $94 \%$ for two nodes, to $62 \%$ for three nodes, and to $33 \%$ for four nodes, what enable us to conclude that even for three concurrent nodes the communication can be done without the RTS/CTS mechanism, assuming that the network is operable for a PDR higher than $50 \%$. On the other hand, if the packet length is 120 bytes, then the PDR for three concurrent nodes becomes $29 \%$, which makes the network operable only for two concurrent nodes. The 120 bytes packets also lead to a higher delay, when compared to the 64 bytes, and 32 bytes packet sizes. The 64 bytes packet size leads to a lower number of transmitted packets what causes less congestion, and, consequently, lower transmission delays, particularly for the 4 nodes scenario.

\section{B. Results for Wi-Fi simulation}

The first simulation setup for Wi-Fi consisted in evaluating the network performance dependency on VWC. Simulations were carried out with 4 nodes generating traffic, and repeated for dry and wet soils. Furthermore, the RTS/CTS mechanism was also studied, and simulations were performed with and without the RTS/CTS mechanism. Fig. 10, 11, and 12 present the results obtained respectively for throughput, PDR, and delay.

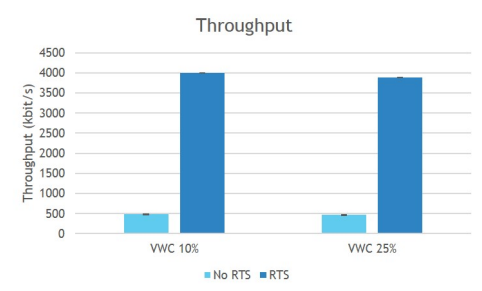

Fig. 10. Throughput for Wi-Fi network for dry and wet soils.

From Fig. 10 and 11 we conclude that the VWC has a negligible effect on the throughput and PDR. On the other 


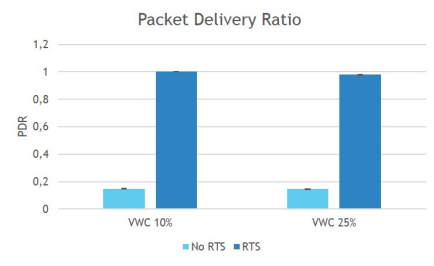

Fig. 11. PDR for Wi-Fi network for dry and wet soils.

hand, the RTS/CTS mechanism has a huge impact on both metrics because with this mechanism enabled the PDR is almost $100 \%$, and the throughput is almost $4 \mathrm{Mbit} / \mathrm{s}$ (the aggregated throughput of the 4 nodes), while with the RTS/CTS mechanism disabled, the PDR drops to $15 \%$, and the throughput to $480 \mathrm{kbit} / \mathrm{s}$.

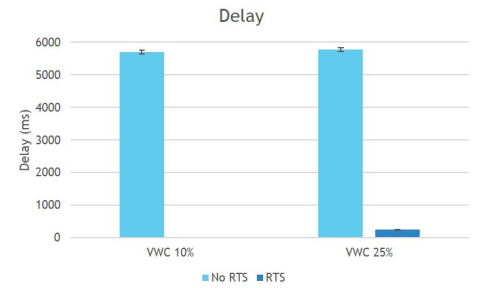

Fig. 12. Delay for Wi-Fi network for dry and wet soils.

Packet delay without RTS/CTS has an increase in 3 orders of magnitude compared to the results with RTS/CTS. This is caused by the fact that the medium access is not done properly, and thus the network becomes saturated. With the network saturated there are several collisions, and consequently several re-transmissions at layer 2 . This associated with the fact that ns-3 re-transmits frames until they reach a 10 seconds delay explains the high delay when RTS/CTS is not used. Also it is possible to conclude that in this case the VWC has a higher impact, because for the RTS/CTS mechanism the delay increases from $2.6 \mathrm{~ms}$ for the dry soil to $239 \mathrm{~ms}$ for the wet soil.

For the second simulation setup the soil was dry, and the purpose of the simulation was to evaluate the network performance for different packet sizes, and for a different number of nodes. Fig. 13, and 14 presents the obtained results respectively for the PDR, and delay.

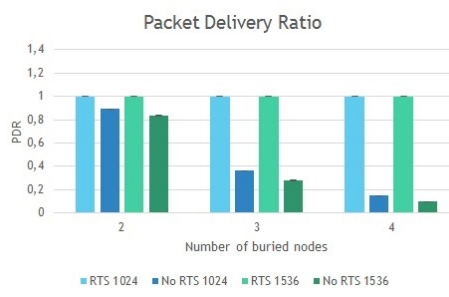

Fig. 13. PDR for Wi-Fi network for dry soil.

From Fig. 13, and Fig. 14 we conclude that the Wi-Fi network can be used without the RTS/CTS mechanism if only two nodes are communicating at the same time. With 3 and 4 nodes the PDR drops below 50\%, and the delay increases significantly, meaning that the network is not operable. It is

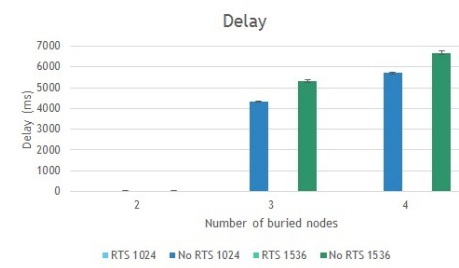

Fig. 14. Delay for Wi-Fi network for dry soil.

also verifiable that higher packet size leads to a higher PDR, and delay.

\section{Results for the positioning system}

In order to collect the RSSi fingerprints a set of 12 points across the terrain was selected. The location of these points is presented in Fig. 3. The results obtained are presented in Fig. 15 and 16 and represent the RSSi fingerprint respectively for a dry, and a wet soils. For the radio map based on the reference points shown in Fig. 3, the average RSSi values, corresponding to each node at each one of the 12 reference points is shown in Fig. 15 and Fig. 16, for dry and wet soil respectively.

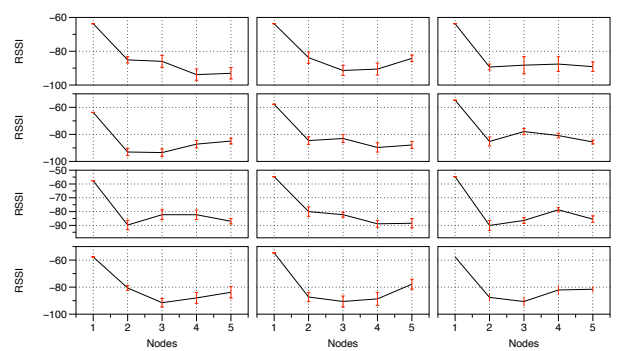

Fig. 15. Fingerprint for Lr-Wpan network for a dry soil.

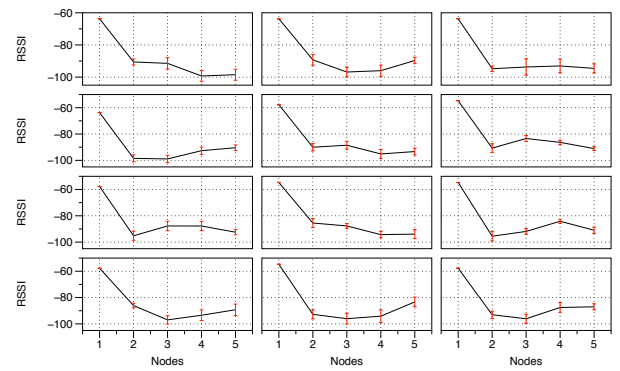

Fig. 16. Fingerprint for Lr-Wpan network for a wet soil.

The main conclusion is that although the RSSi values drop for the wet soil, what was expected due to the higher attenuation introduced by water, the RSSi keeps the same "shape" or "fingerprint" from one soil condition to the other. This fact is important, because it enables a user to collect RSSi samples to build a radio map, and then use that radio map without considering the soil humidity.

1) Results for localization: In order to evaluate the accuracy of the radio maps for localization we collected the samples to build two radio maps (configurations in Fig. 3 and Fig. 4 for dry soil; then we collected two sets of test samples randomly placed across the simulation area, one for dry soil and another 
TABLE II. LOCALIZATION ERROR.

\begin{tabular}{|c|c|c|c|c|c|c|}
\hline \multirow[t]{2}{*}{ Simulation } & \multicolumn{2}{|c|}{25 points (dry) } & \multicolumn{2}{|c|}{12 points (dry) } & \multicolumn{2}{|c|}{25 points (wet) } \\
\hline & Precision (\%) & Error (m) & Precision $(\%)$ & Error (m) & Precision $(\%)$ & Error $(\mathrm{m})$ \\
\hline 1 & 90 & 7.3 & 66.7 & 9.0 & 20 & 17.3 \\
\hline 2 & 83 & 6.3 & 53.3 & 8.5 & 13.3 & 16.7 \\
\hline 3 & 93.3 & 6.3 & 70 & 8.6 & 26.7 & 16.0 \\
\hline 4 & 80 & 7.4 & 66.7 & 8.8 & 16.7 & 16.4 \\
\hline
\end{tabular}

for wet soil. Finally, we estimated the position of the dry test samples using the two radio maps, and estimated the position of the wet test samples using one of the radio maps (the one with 25 reference points that was built for dry soil).

The precision measurement was calculated based on the nearest known fingerprint. For each test sample we computed the similarity (Euclidean distance) with each fingerprint in the radio map, and selected as the nearest neighbour the fingerprint corresponding to the shortest Euclidean distance. If the nearest point estimated based on this method is in fact the real nearest point we consider that the right position was estimated, otherwise we consider an error. The number of correct guesses divided by the total number of random points gives the precision reported in Table II. The average error reported in Table II was calculated as the average of the euclidean distances between each exact position of each test sample and the position of the nearest point estimated.

From the results presented in Table II we conclude that the localization system has a high accuracy for the grid map when the soil is dry (accuracy above $80 \%$ ). When the number of measured points is reduced, the accuracy drops to a minimum value of $53 \%$, and the position error increases to a maximum of $9 \mathrm{~m}$, which is expected since the measured points are now more sparse. For a wet soil the accuracy drops abruptly because the radio map was designed to a dry soil. The Euclidean distance was used for comparing points but, as demonstrated in Section IV-C, a more sophisticated metric based on the RSSi shape of the 5 points could be used to improve the precision and reduce the average error.

\section{CONCLUSION}

This work evaluated the performance of the multiple access in WUN for both Lr-Wpan, and Wi-Fi standards, and for dry and wet soils. We conclude that the RTS/CTS mechanism has a major impact in the medium access, much higher than the water content. In fact, for four concurrent nodes we verified that the usage of the RTS/CTS mechanism enabled the packet delivery ratio to increase from $15 \%$ to almost $100 \%$. For 2 concurrent nodes the network can work effectively without this mechanism and avoid unnecessary overhead. These simulations allowed us to conclude that the RTS/CTS mechanism is suitable for WUN.

The evaluation of the positioning system at the soil surface was done for the Lr-Wpan network. Based on a radio map with 12 reference points, we conclude that the RSSi drops for the wet soil, when compared to the dry soil, but it keeps similar fingerprints what makes the radio map suitable to different soil conditions. A simple localization system was proposed that lead to precision values ranging from $80 \%$ to $90 \%$, and an average positioning error of $7.4 \mathrm{~m}$. This average error increases to $9.0 \mathrm{~m}$ if instead of a radio map with 25 points is used a map with 12 points. The average error is further increased to $17 \mathrm{~m}$ if the radio map for dry soil is applied on a wet soil.

\section{ACKNOWLEDGMENT}

This work has been partially supported by COMPETE: POCI-01-0145-FEDER-007043 and FCT - Fundação para a Ciência e Tecnologia within the Project Scope: UID/CEC/00319/2013. This work is financed by the ERDF - European Regional Development Fund through the Operational Programme for Competitiveness and Internationalisation - COMPETE 2020 Programme within project «POCI-010145-FEDER-006961», and by National Funds through the FCT - Fundação para a Ciência e a Tecnologia (Portuguese Foundation for Science and Technology) as part of project UID/EEA/50014/2013. The first and second authors would like to thank the support from the Portuguese Foundation for Science and Technology (FCT) under the fellowships $\mathrm{PD} / \mathrm{BD} / 105861 / 2014$, and PD/BD/105865/2014.

\section{REFERENCES}

[1] S. Conceição, F. Ribeiro, R. Campos, and M. Ricardo. A ns-3 based Simulator of TCP / IP Wireless Underground Networks, in Proc of Wireless Days 2014, 2014.

[2] W. Xiao, W. Ni, and Y. K. Toh. Integrated Wi-Fi Fingerprinting and Inertial Sensing for Indoor Positioning, in Proc of Indoor Positioning and Indoor Navigation (IPIN), 2011

[3] X. Dong, M. C.Vuran, and S. Irmak, Autonomous precision agriculture through integration of wireless underground sensor networks with center pivot irrigation systems. Ad Hoc Networks, 1975-1987, 2013.

[4] Z. Sun, P. Wanga, M. C. Vuran, M. A. Al-Rodhaan, A. M. Al-Dhelaan, and I. F. Akyildiz. BorderSense: Border Patrol Through Advanced Wireless Sensor Networks. Ad Hoc Networks, page 468-477, 2011.

[5] T. AL-Kadi, Z. AL-Tuwaijri, and A. AL-Omran, Wireless sensor networks for leakage detection in underground pipelines: A survey paper, in Proc. of AASNET, 491-498, 2013.

[6] X. Dong and M. C. Vuran. A channel model for wireless underground sensor networks using lateral waves, in Proc. of GLOBECOM, 2011.

[7] Z. Sun, I. F. Akyildiz, and G. P. Hancke. Dynamic connectivity in wireless underground sensor networks. Wireless Communications. IEEE Transactions on Wireless Communications, 10(12):4334 - 4344, 2011.

[8] X. Dong, and M. C. Vuran. Empirical Analysis of the Hidden Terminal Problem in Wireless Underground Sensor Networks, in Proc of ICWCUCA 2012, 2012.

[9] H. Xiaoya, G. Chao, W. Bingwen, and X. Wei. Channel Modeling for Wireless Underground Sensor Networks. IEEE 36th Annual Computer Software and Applications Conference Workshops, 2011.

[10] P. M. Shankar. "Fading and Shadowing in Wireless Systems", ISBN-13: 978-1461403661, Springer 2012

[11] S. Conceição, F. Ribeiro, R. Campos, and M. Ricardo. Novel ns-3 model enabling simulation of electromagnetic wireless underground networks, in Proc of WNS3 '15, 2015. 